\title{
Evaluation Of The Integrated Care Model: Child Morbidity Reduction In Mashonaland East, Zimbabwe
}

\author{
M. Mhlanga
}

\begin{abstract}
Zimbabwe has one of the highest prevalence rates on preventable child morbidity in the world. This is mainly attributable to the absence of an effective community health mobilisation structure that reaches all targeted households with correct and consistent social behaviour change interventions for better child and maternal health.

To address this, a cluster randomised controlled trial was conducted to assess the effectiveness of a developed integrated community intervention approach in reducing child morbidity and improving maternal health outcomes. A total of 765 motherchild pairs (413 in the intervention and 352 in the control) from 2 districts in Mashonaland East province were recruited and followed up for 12 months. Only women with children aged 0 48 months at the beginning of the study were selected. Participants were selected (and recruited) through stratified random sampling from 30 villages/clusters $(16$ in the control and 14 in the intervention) out of the total of 43 villages in the 2 districts. The intervention arm received education on maternal and child health through an Integrated Care Model mobilisation system whereas participants in the control arm were mobilized and educated using the conventional mobilisation system. Baseline and end-line surveys were done to assess and compare baseline characteristics and secondary study outcomes. The primary outcome was child morbidity in the follow-up period of 12 months.
\end{abstract}

The mean age of participating mothers was 28 years $(\mathrm{SD}=$ 6.8) and that of participating children was 18.2 months $(\mathrm{SD}=$ 4.0). The risk of child morbidity was $37.5 \%$ in the control and $22.0 \%$ in the intervention representing a relative risk of 1.7 [95\% C.I (1.4-2.1)]. The incidence rate of child morbidity was 0.043 and 0.022 episodes per child year in the control and intervention arm respectively giving an incidence rate ratio of $2.0(p<0.001)$. This ratio meant that the chance of being a disease case in the control was double that in the intervention arm. Women in the intervention arm had statistically significant $(p<0.001)$ higher knowledge about maternal and child health and better child care practices at the end of the study.

There was strong evidence that the Integrated Care Model did not only reduce child morbidity but also improved maternal knowledge, health-seeking behaviour and care practices. Accordingly, governments in developing countries and countries in poor resource settings could strengthen their community health delivery systems by implementing this low-cost, sustainable and high-impact approach.

Index Terms - Child morbidity, Integrated Care Model, Community mobilization.

\section{INTRODUCTION}

Perinatal conditions contribute significantly to child morbidity in developing countries. According to 2015 statistics, 11 million children under five die each year, 4 million of them in the neonatal period and $98 \%$ in developing countries with $64 \%$ of them due to preventable causes [1]. Global statistics in 2015 revealed that pneumonia, diarrhoea and malaria are the major childhood illnesses responsible for child death in Africa, accounting for one third of the global under-five mortality and $40 \%$ of deaths of children under five in Sub-Saharan Africa [2].

Zimbabwe is one of the countries in Sub-Saharan Africa that has seen stagnancy and reversals in survival rates due to preventable child morbidities such as diarrhoea, acute respiratory infections and malaria which are the leading forms of morbidity in childhood. The country has 10 provinces and of these, Mashonaland East province significantly accounts for the high levels of child morbidity, being in the top 3 provinces with Mashonaland Central and Manicaland province.

Effective community mobilisation to promote maternal and child health is critical in reducing child morbidity. An effective community mobilisation approach should reach all households with key health education and promotion information and should effectively promote and monitor caregivers' knowledge about maternal and child health, healthcare-seeking behaviour and childcare practices continuously in all house-holds in a targeted community.

The absence of an effective integrated community mobilisation approach to promote maternal and child health has resulted in the high prevalence of preventable child morbidity and mortality in Zimbabwe. The main focus in Maternal Neonatal and Child Health (MNCH) has been on strengthening facility-based health systems yet about $74 \%$ of maternal and child deaths are occurring at community level (MoHCC, 2009). The Sustainable Development Goal number three (SDG 3) advocates for Universal Health Coverage and reduction of under-five mortality rate to less than $25 / 1000$ live-births by 2030 through effective community mobilisation [3]. To attain this target, efforts in community mobilisation have to be multiplied more than 4 times in Mashonaland East.

Piecemeal, fragmented and unsustainable interventions have been implemented in varying degrees from one province to another and this has been ineffective in the reduction of child morbidity since the mobilisation approaches were not mainstreamed into the health delivery system. The Government of Zimbabwe is currently implementing the results-based financing programme that is funded through the World Bank and the health transition fund, in a bid to increase access and utilisation of MNCH services across the country. This however had little impact in influencing health-seeking 
behaviours since it's only implemented at facility level and only motivates health professionals to provide quality services in anticipation of subsidies that they get for successful fulfillment of targeted health outputs.

Health promotion and health-seeking behaviour can only be effectively addressed and measured by saturation of interventions at community level addressing the root causes of poor health- seeking behaviours and measuring the effect of intervention. The current conventional community mobilisation and health delivery system in Zimbabwe heavily relies on the existence of one cadre, the Village Health Worker (VHW) at community level. Each village has a VHW, who is supposed to receive 8 weeks training on community health especially primary healthcare. The average size of each village ranges between 150 and 600 households depending on the terrain of the given place and settlement pattern.

The VHW is a part time volunteer, who is given an allowance of 10-15 dollars every month for the service they offer to the community. The VHW reports to the local health centre every month and collects community vital statistics and health data on various health indicators. They use opportune moments and events to raise health awareness in places like funerals or in village meetings that the kraal head or headmen would have called and they are usually given 10 to 20 minutes to talk about any health matters they may deem necessary hence they use the 'supermarket approach' to raise awareness and conduct health education. This means that most of the health education sessions are not really planned and they are ad hoc. VHWs are expected to do home visits and follow-ups of all the households in their village(s) on different aspects of health such as Prevention of Mother to Child Transmission of HIV (PMTCT), Tuberculosis (TB) contacts, pregnant women, women post-delivery, disease surveillance among other things.

However, due to the high costs of training and equipping one VHW, the high attrition rate and mobility of these community health workers, the coverage on the Village Health Worker programme is far below optimal and most districts barely have $50 \%$ of their villages managed by a fully trained VHW. This means that nearly $50 \%$ of the communities in rural Zimbabwe hardly get consistent community health education and promotion programmes to prevent and reduce child morbidity resulting in high incidence of child morbidity.

Globally, financial investors in the health sector are advocating for results-based financing, which in some circles is also referred to as performance-based financing. This aims at ensuring that there is both cost-effectiveness and program effectiveness in a bid to ensure that there is value for money for all the investment efforts.

The primary healthcare approach dictates that there should be a hundred percent coverage of all communities and households with preventive, promotive and rehabilitative interventions. Sustainable development goal number 3 dictates that health services should reach everyone, everywhere regardless of their race, age and ethnicity with an aim of eradicating preventable neonatal and under-five morbidity and mortality rate. It is then prudent that a model be designed that meets both the dictates of quality and demand (coverage) in the community health service delivery to reduce preventable morbidity and mortality in Zimbabwe since none exists to date.

Zimbabwe is a developing country, hence like any other developing country suffers economic challenges and resource constraints in health delivery. Very few programmes have also taken an initiative to promote high accountability and meaningful participation of communities in their own health. As a result, some novel innovations have not achieved optimal results because they were heavily managed and influenced by a greater proportion of external staff and for a fixed time period.

There is a greater chance of survival and effectiveness if a community mobilisation approach is fully integrated into the mainstream health delivery structure as opposed to stand alone programmes. This study determined the effectiveness of an Integrated Care Model (ICM) approach in reducing child morbidity in Mashonaland East, Zimbabwe using a cluster randomised controlled trial. This research design was the best for the study since it made it possible to determine the effect of the innovative intervention on child morbidity by comparing the intervention and the conventional mobilisation system that exists in Zimbabwe.

The study sought to provide health policymakers with new evidence-based, integrated options for strategies to effectively reduce community child morbidity and reduce preventable child mortality

\section{A. Hypothesis}

The Integrated Care Model (ICM) will be associated with a reduction in child morbidity.

\section{B. Research objectives}

1. To determine the incidence of child morbidity in the intervention and control arm in 12 months of follow-up

2. To determine the risk of child morbidity in the intervention and control arm in the 12 months of follow-up

3 . To determine the proportion of severe and repeat cases of pneumonia, diarrhoea, malaria, malnutrition, fever and anaemia in intervention and control at the end of the study follow-Up.

\section{METHOD}

\section{A. Study design and ethical approval}

A cluster randomised controlled trial was used since the unit of randomization was the village rather than the individual mother and child. The study had two arms, the intervention arm where the ICM model was implemented and the control arm which basically implemented the conventional community mobilisation system for Zimbabwe. Villages were matched and randomly assigned to either the intervention arm or the conventional arm. All the recruited participants (mother-child pairs) underwent a baseline assessment and were prospectively followed up for 12 months. At the end of the study, an endline survey was conducted to determine the effect of the intervention on study secondary outcomes. The results at the end of the follow-up period determined whether the intervention significantly reduced child morbidity compared to the conventional mobilisation approach.

The study was conducted in line with ethical principles enshrined in the Declaration of Helsinki. Permission to 
conduct the study was obtained from the Joint Research Ethics Committee (JREC), approval number JREC/86/16 and the Medical Research Council of Zimbabwe (MRCZ), approval number A/2099. The JREC and MRCZ reviewed all documentation to safe guard rights, safety and well-being of participants. All case report forms, study reports and communication identified participants by assigned trial codes. The investigator conducted the study in compliance with the approved protocol and no adverse effects were reported.

\section{B. Study setting and population}

The study was conducted in two rural districts of Mashonaland East province in Zimbabwe namely Murewa and Seke. Mashonaland East is in the top 3 provinces with the highest child morbidity and mortality rates in Zimbabwe (NNR 38/1000 and U5MR 97/1000) (MICS, 2014). Figure 1 below shows the map of Mashonaland East province and the shaded districts Seke and Murehwa were the selected districts in this study with Goromonzi and Marondera being the buffering districts between.

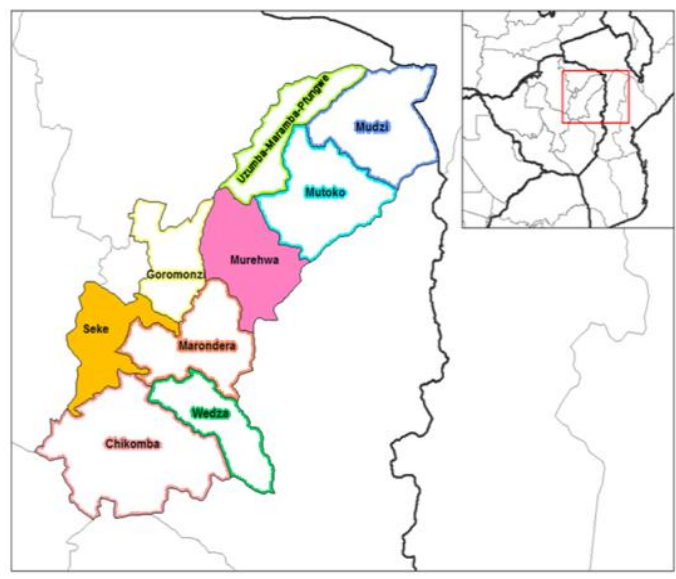

Fig. 1. Study sites.

Mashonaland East has a total population of 1371989 people and of these $23.7 \%$ (324 543) are women of childbearing age and $209579(15.3 \%)$ are children below 5 years of age [4], [5]. Women of child-bearing age (15-49 years) made up the study population and the study targeted pregnant women and women with children 0-48 months in the selected clusters.

\section{Sample size determination}

The trial sample size was calculated in STATA 15 software, assuming child morbidity prevalence of $50 \%$ with a margin of error of $5 \%$ to achieve power of 0.9 . The assumed intra-cluster correlation coefficient (ICC) was 0.05 and the level of significance was $5 \%$. A design effect of 2.45 was factored in the sample size determination to counter for possible bias due to clustering. Firstly, assuming individual randomization, the sample size per arm would be 121 participants.

After allowing for cluster randomization, the calculated sample size per arm was 330 mother-child pairs. The calculated number of clusters per arm (m) was 11 . The study however recruited 765 mother-child pairs, factoring in an attrition rate of $10 \%$. The study recruited all consenting women with children 0 to 48 months staying within the selected clusters in both the intervention and the control arm. There were 413 mother-child pairs in the intervention arm and 352 mother-child pairs in the control. Women who did not dwell permanently in the study clusters with their children were excluded and so were very ill women and mentally challenged women.

The minimum sample size for the endline survey was determined using Dobson's formula. Assuming that $\mathrm{z}$, standardized normal distribution value for the $95 \%$ CI, which is $1.96, p=0.5$, an estimate of prevalence of child morbidity in Mashonaland East and taking $\Delta$, the margin of error to be 5.0\%. $n=z 2 p(1-p) / \Delta 2=(1.96) 2(0.5)(0.5) /(0.05) 2=384$.

In each arm a total of 192 women who had participated in the study from its inception were interviewed by the research assistants. Simple random sampling using a hat with cards either labelled 'yes' or 'no' was used to select participating women for the endline survey.

\section{Sampling and recruitment procedure}

The Fig. 2 outlines the sampling procedure and the stages for participant recruitment at study inception.

SAMPLING FRAME: Heavily burdened comparable districts (child morbidity) - identified through review of baseline characteristics from the provincial data base of child indicators (2014-2016) -

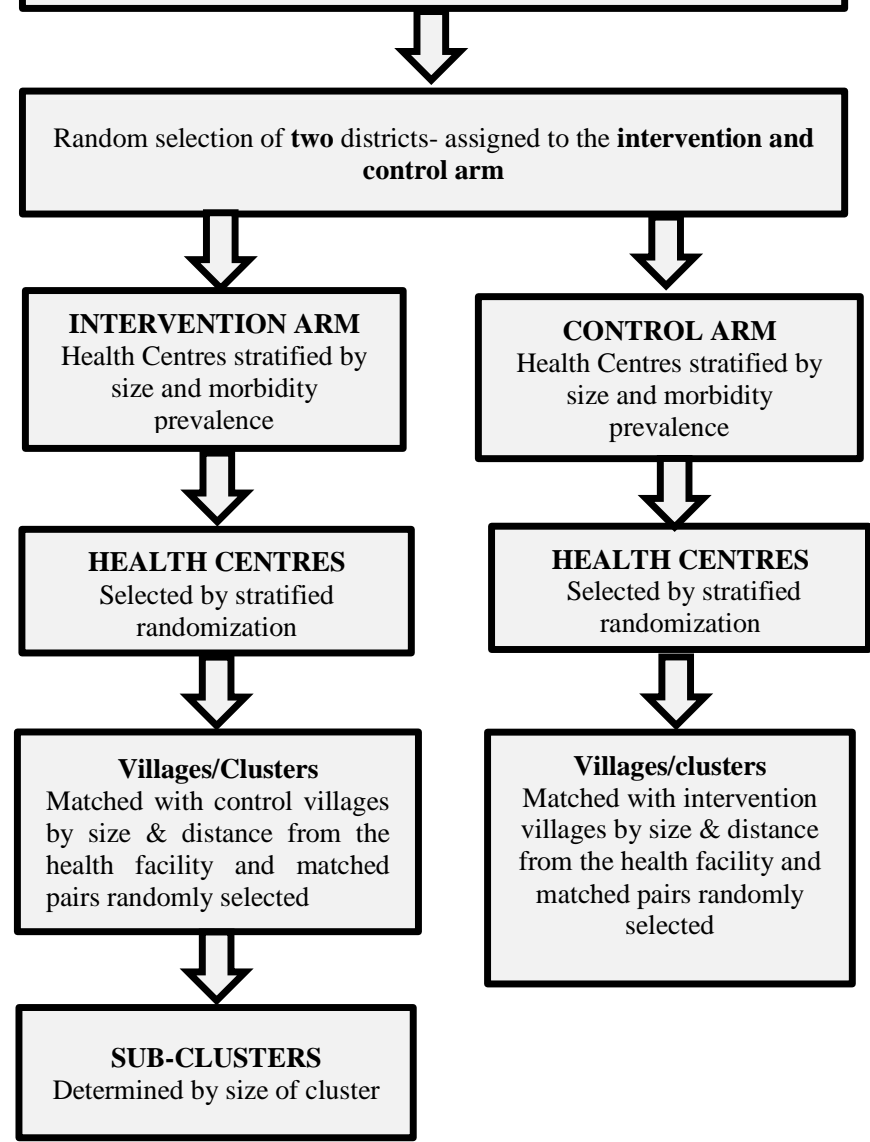

Fig. 1. Sampling procedure.

A review of the provincial data base on child health indicators was conducted to identify and select the districts with highest burden on child morbidity. The review considered cumulative number of cases of children (0-59 months) who had pneumonia, diarrhoea, malaria, anaemia 
and acute malnutrition for a 2-year period between October 2014 and October 2016.

Districts with the highest morbidity constituted the sampling frame and from these, 2 districts were selected by simple random sampling, picking cards from an elevated box which contained the names of the potential district. The first district was assigned to the control arm and the second district to the intervention arm. Health centres from the selected districts were stratified by size and child morbidity prevalence to reduce imbalances.

Health centres were ranked in their respective districts by the cumulative number of cases of child morbidity (October 2014 to October 2016) and categorized into thirds and the first third represented health centres with the highest morbidity, the second third represented health centres with medium morbidity and the last third represented low morbidity. Health centres which fell in the high and medium category according to the criteria above constituted the sampling frame for health centres and from these matched pairs of health centres we then randomised the health centres either to the intervention or control arm accordingly.

Villages were the targeted clusters. A village in Zimbabwe has an average population size of 500. A list of all villages from the selected health centres was compiled and the villages were matched on two demographic characteristics namely village size (a proxy indicator of population density) and geographical proximity to the health centre.

Central randomization was employed using the computergenerated sequences generated by an independent analyst and an independent researcher who was not part of the research team randomised the clusters. Matched pairs of clusters were then randomised to either the control or intervention arm accordingly until the minimum number of clusters needed per arm as determined from the sample size calculation was reached. Mother-child pair recruitment considered all eligible participants in the selected clusters to reduce the risk of recruitment bias.

A questionnaire was used to obtain baseline data. It had questions on the following demographic data: maternal age, age of the household head (HH), child age, birth-weight of the child, gestational age at delivery, number of children in each family, sex of the household head $(\mathrm{HH})$, education level of the respondent, their marital status, religion and major source of health education. The questionnaire used for baseline assessment was assessed for content validity against set objectives by 4 independent content experts from the University of Zimbabwe. The experts were requested to assess the relevance of each question/item in the instrument on a 4- point scale.

The questionnaire was evaluated for test-retest reliability with 10 people randomly selected at Kunaka rural health centre who were from non-participating villages. The testretest assessments were 5 days apart. Participants were not told that they would be re-tested to minimise bias. Item completion of the questions and percentage agreement between test-retest assessments was calculated for each question and it was in the range of $90-100 \%$ for all questions. Based on these results the questionnaire was adopted for use in the study.

Independent data collectors were employed and trained on how to collect baseline survey data at recruitment. Data collectors for baseline data were blinded to minimise participant or observer bias. Baseline data was collected between November 2016 and end of March 2017 and this period coincided with the study recruitment period. Following the baseline survey, key stakeholders in Ministry of Health and the University were engaged for the finalisation of the intervention model (ICG model).

\section{E. Description of the interventions in study arms}

\section{Description of the intervention arm processes}

A training manual was developed and used to train both VHWs and their Community Groups (CGs). Each VHW in the intervention group received a five days' training on how to facilitate $\mathrm{CG}$ sessions using participatory approaches and how to monitor CGs. The same training sessions oriented the VHWs to the community data collection tools that they would use for monthly data collection. A nurse who has been trained on the integrated management of childhood illnesses at each clinic was part of the VHW training program to enhance sustainability and ownership.

This focal nurse also received training on how to monitor and coordinate the VHWs and CGs activity in line with the community integrated prevention and management of childhood illnesses guidelines. The nurse had an overall responsibility of giving feedback to the community and updates on new guidelines on childhood sickness management hence there was a continuous two-way communication between the communities in the clinic's catchment area and the health centre mediated by the VHWs. The nurse was also trained on how to collect monthly facilitybased data on child morbidity and how to use codes to report on new cases of child morbidity using the health facility tool.

A clinic covered at least 8 villages in its catchment area. A CG was established in each intervention cluster (village) and this comprised of 4-12 members who were volunteers from each respective village in the intervention arm. A village in Zimbabwe has an average size of 150 households hence the average number of volunteer members in each CG was 10. Each Volunteer Group Leader (VGL) was a caregiver of at least one child who is 0 to 48 months old and was selected by the members from a cohort (sub-clusters) of 10 households in their locality, whom they represented in the CG. This was done with the full support and buy-in of local traditional leadership (Headmen).

Each CG was facilitated by a registered VHW who had received the 5-day training on CGs. Facilitation of health education sessions at sub-cluster level (Household cohort level) was done by each respective VGL. VGLs received oneday orientation training on the mobilisation approach and their expected roles and responsibility. The traditional leaders supported the CG activities, reinforced their health messages and worked with groups to take wider action on community health issues.

Volunteer Group Leaders reported to the VHW who in turn reported to the nurses at their local clinic. This empowered the VHWs and strengthened their role, confidence, capacity and support as community health workers. CG sessions commenced in each village with each session focusing on one or two thematic areas in maternal and child health care and running for 1- 2 hours only. Educative sessions continued for 
12 months. Sessions were conducted at least once every fortnight at CG level by a trained VHW.

Each VGL would in turn replicate the same session(s) in his/her respective cohort of 10 households each fortnight for the same duration in a participatory manner. The participants shared their experiences and challenges in pregnancy, delivery, post- delivery and childcare and these were recorded respectively.

The manual used for $\mathrm{CG}$ and cluster facilitation covered lessons on $\mathrm{MNCH}$ along the continuum of care (care during pregnancy, delivery, postnatal care and community integrated management of childhood illnesses). Participatory approaches were used to facilitate learning at the community level. These included: role plays; simulations, demonstrations, group discussions, testimonials, case studies, photo galleries, community mapping and pocket charting. Edutainment approaches such as drama, songs, storytelling (including stories with a gap) and problem tree analysis were used to facilitate some of the sessions for health education on bad and recommended maternal and child health practices.

Principles of the 'open space technology' were incorporated in some sessions to allow for full, active participation of all members and enhance accountability in the matters relating to their own health. VGLs primarily targeted care-givers of children 0- 59 months and pregnant women in their clusters of 10 households though other people were free to join sessions. The VGLs conducted at least two home visits each month to monitor health practices and behaviours and also provide support and individualized health education in targeted households. VGLs compiled reports and statistics on child and maternal health indicators monthly. The VHW would then consolidate the statistics from each VGL and compile a monthly report which he/she submitted to the clinic monthly.

When a member of a CG dropped out, reasons for dropping would be ascertained and documented. The beneficiary mothers would select another person from their group to replace the member who would have left. Contact with the community was assessed through signed attendance registers and periodic monitoring and supportive supervisions.

Community-integrated management of childhood illness topics included good health care, community prevention and management of diarrhoea, good childcare, expanded program on immunisation, importance of regular growth monitoring, prevention and management of malaria. Other topics were on prevention of pneumonia and danger signs, when to seek healthcare services at the health facility and importance of community health workers in child health.

\section{Description of the control arm processes}

In the control arm, the conventional system was implemented and monitored. The VHWs in the control clusters and their respective focal nurses were oriented on the study. They only received further training on the use of the monthly data collection forms. VHWs continued their day to day routine activities according to the guidelines of the Ministry of Health and Child Care and reported to their nearest clinic. The Ministry of Health guidelines stipulate that VHWs should be able to carry out all the 14 elements of the Primary Health approach at village level and report their activities monthly to their nearest clinic.

\section{F. Intervention-monitoring parameters}

Intervention dosage in community and public health refers to the number of activities implemented or number of programs received by the intended population. This was measured by indicators such as clinic attendance, number of community mobilisation meetings and the number of home visits. The program's effect is a function of the number of doses given to the recipients. This intervention was designed and ran for 12 months in the intervention clusters.

Programme specificity refers to the specific intervention activities that are implemented to address the objectives of the program. The study developed a community training manual to promote child health by preventing and early management of diarrhoea, malaria, pneumonia, fever and malnutrition through specific intervention activities for each targeted illness.

Intervention intensity refers to an optimal level at which educative sessions are expected to be delivered per month. The intervention package had a total of 22 planned educative sessions (15 focusing on the primary outcome and 7 on the secondary outcome(s)). On average a CG and its respective sub-clusters (household cohorts) were expected to conduct 46 educational sessions per month. Each session was expected to last for at least 30 minutes. The expected minimum standard frequency of meeting was once per fortnight with each meeting lasting for an average of 2 hours. Sessions on the primary outcome were expected to be completed within 3 months and those on secondary outcomes within 1.5 months. The intervention package was expected to have $2-3$ repeat doses during the period of study follow-up both at cluster and sub-cluster level in the intervention arm.

Priority was given to child health outcomes and the most prevalent child health issues were prioritized from cluster to cluster. VHWs and their respective VGLs were expected to each conduct at least 3 home visits per month targeting households with either a pregnant woman or a mother with a child below five years. Educative sessions in home visits were expected to be target specific and tailor made to address focused problems or knowledge gaps elicited during an interaction with a caregiver for a particular household.

Post-randomization recruitment bias was managed by including objective measures of eligibility, and routine frequent checking for differences between arms in numbers of recruits and refusals. All the mother-child pairs that were recruited for this study were coded for the sake of identity concealment and privacy. Follow-ups and on-job mentorship were conducted to CGs quarterly to assess fidelity of training and acceptability of the intervention strategies in the intervention arm. VHWs were monitored quarterly in both arms and participants were followed-up for the twelve-month period.

\section{G. Data quality management and analysis}

Data was collected at baseline, during follow-up period and through an endline survey at the end of 12 months of intervention implementation. A standard health facility data collection form was used by the nurses in the participating clinics to collect monthly data on child morbidity for children who had been recruited in the study.

Cases of pneumonia, diarrhoea, malnutrition, fever, anaemia and malaria were reported each month using 
assigned codes for mother-child pairs and entered in STATA 15 for analysis. Severity of disease conditions was classified using the recent international IMNCI guidelines for 2015.The trial's primary endpoint was child morbidity during 12 months of intervention. Additional endpoints included home care practices and health care-seeking in the antenatal, delivery and postnatal period. The effect of the intervention was measured through a prospective surveillance system. In both intervention and control villages, all morbidities of U5s were recorded monthly. Records of a woman's migrations within and outside of the study area were recorded in order to allow for both 'intention to treat' and 'per protocol' analysis. Validity of data was ensured through the prevention and management of transcription errors in data entry. Data was checked for completeness, timeliness and precision by both the investigator and the data analyst.

Data manipulation was also kept in check through records review in all the sites and random reviews of data entered in the computer data base against the hard copies to check for accuracy. The study established information systems with consistent protocols and procedures. Standardized, written instructions for data collection were given to data collectors in study sites.

The unit of randomization was the cluster whereas the units of inference were individuals within the clusters who met the inclusion criteria. Intention to treat analysis was used in data analysis.

The effectiveness of the intervention was assessed using measures such as incidences, risk difference, odds ratio, timeto-event analysis and difference-in-difference analysis. Differences between groups were assessed for statistical significance using chi-squared tests and t-tests. Logistic regression was also employed to assess the differential effect of the following predictors on child morbidity: age of caregiver, religion, knowledge about childcare, childcare practices and health-seeking behaviour.

\section{RESULTS}

\section{A. Participant flow}

The first participant was recruited on 28 November 2016 and the last participant was recruited on 20 March 2017. The follow-up phase ended on the 31st of December 2017. The two districts that participated had a total of 45 rural health centres (Murewa (26), Seke (19)). Health centres were stratified and matched according to the total number of cases of childhood illnesses that were recorded between January and October 2016. Villages (clusters) were matched on two demographic characteristics namely village size (a proxy indicator of the population density) and geographical proximity in relation to the nearest health centre. Matched clusters were then randomised to either the control or intervention arm accordingly.

The two-participating health centres in the control arm namely Marirangwe clinic and Kunaka clinic had 10 clusters (175 mother-child pairs) and 6 clusters (177 mother childpairs) respectively at the end of the recruitment phase and study end-point. In the intervention arm, the two-participating health centres namely Murewa and Macheke had 9 clusters (260 mother-child pairs) and 5 clusters (153 mother-child pairs) respectively.

Fig. 3 illustrates the flow of clusters and individual participants through each stage of the study, showing for each arm, the numbers of clusters. The figure also highlights those who received the intended treatment, completed the study as per protocol, and got analysed for the primary outcome.
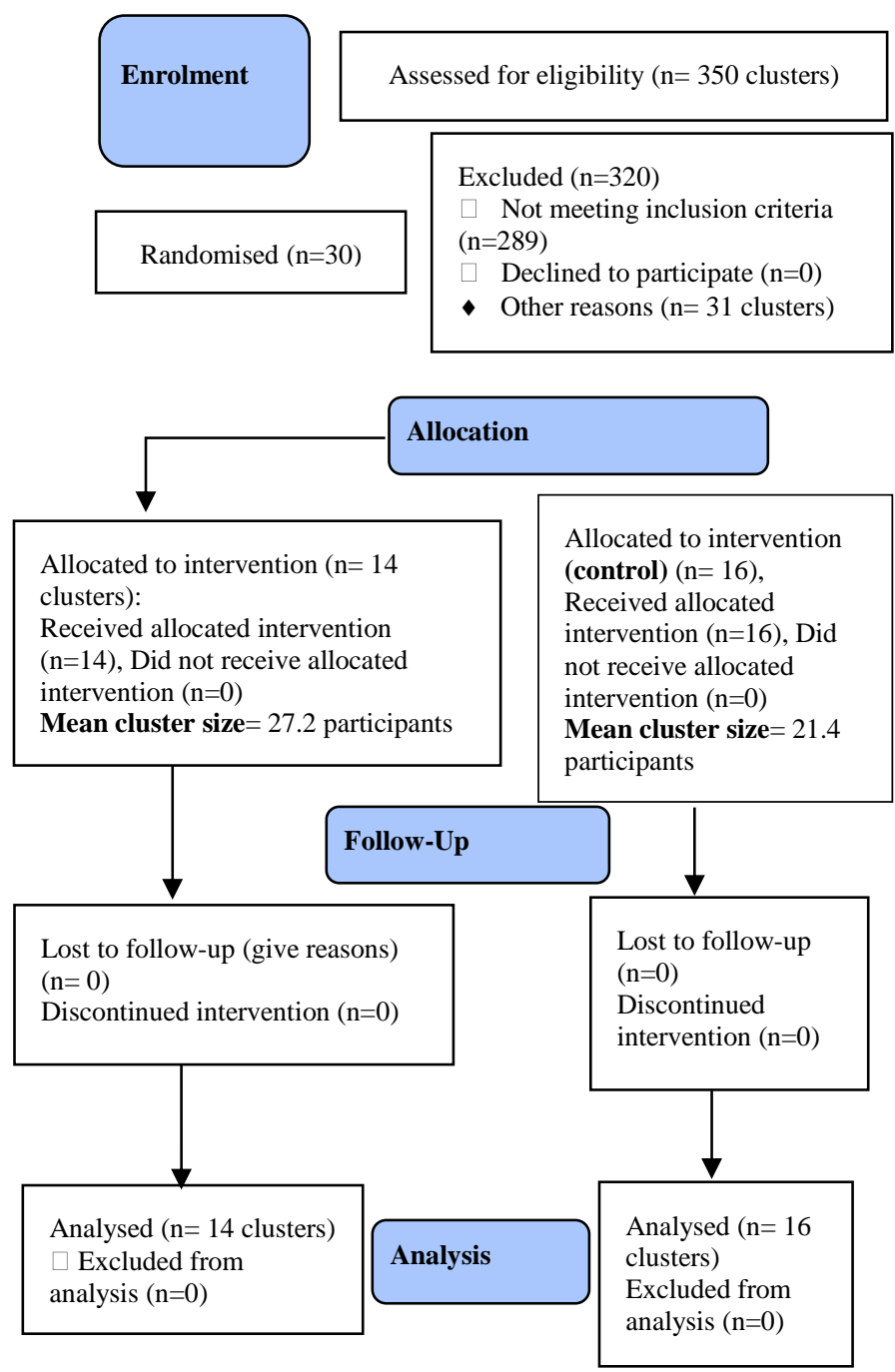

Fig. 3. Flow diagram for the study clusters.

\section{B. Socio-demographic characteristics of the study arms}

A total of 672 women with children aged 0-48 months were interviewed. The mothers' mean age was 28.0 years ( $\mathrm{SD}=6.8)$, 95\% C.I (27.4 - 28.5). The children's ages followed a normal distribution with a mean of 18.2 months and a standard deviation of $4.095 \%$ C.I (17.2-19.3). The mean gestational age at delivery for the participating mothers was 39 weeks $(\mathrm{SD}=1.8)$. The mean number of children for the participating women was $2.6(\mathrm{SD}=1.5)$. Household heads had a median age of 33.5 years $(Q 1=25 ; Q 3=94)$. The mean length of exclusive breastfeeding was 5.8 months $(\mathrm{SD}=0.1)$, 95\%C.I. (5.6- 5.9) and the mean frequency of child feeding was 4.8 times $(\mathrm{SD}=0.14), 95 \%$ C.I (4.5-5.1). Table 1 below summarises the socio-demographic results from the survey. 
TABLE 1: DEMOGRAPHIC CHARACTERISTICS OF STUDY ARMS (N=672)

\begin{tabular}{|c|c|c|}
\hline Variable & Intervention & Control \\
\hline $\mathrm{N}=672$ & $\begin{array}{c}379 \\
(56.4 \%)\end{array}$ & $\begin{array}{c}293 \\
(43.6 \%) \\
\end{array}$ \\
\hline Mean Mothers' age(years) & $\begin{array}{c}27.8 \\
\text { SD 7.1 }\end{array}$ & $\begin{array}{c}28.2 \\
\text { SD } 6.5\end{array}$ \\
\hline Mean Child age(months) & $\begin{array}{c}17.8 \\
\text { SD13.8 }\end{array}$ & $\begin{array}{c}18.8 \\
\text { SD14.4 }\end{array}$ \\
\hline Mean birth weight $(g)$ & $\begin{array}{c}3019.4 \\
\text { SD } 503.9 \\
\end{array}$ & $\begin{array}{c}3114.8 \\
\text { SD573.8 } \\
\end{array}$ \\
\hline Mean age of $H H$ (years) & $\begin{array}{c}32.8 \\
\text { SD } 13.5 \\
\end{array}$ & $\begin{array}{c}34.3 \\
\text { SD } 11.4\end{array}$ \\
\hline Mean gestational age(weeks) & $\begin{array}{c}39.7 \\
\text { SD1.5 }\end{array}$ & $\begin{array}{c}38.6 \\
\text { SD } 1.9\end{array}$ \\
\hline $\begin{array}{l}\text { Sex of } H H \\
\text { Male }(\%) \\
\text { Female }(\%)\end{array}$ & $\begin{array}{c}309(81.5) \\
73(19.3)\end{array}$ & $\begin{array}{c}259(88.3) \\
34(11.6)\end{array}$ \\
\hline $\begin{array}{l}\text { Number of children per } \mathrm{HH} \\
<4(\%) \\
4 \text { and above }(\%)\end{array}$ & $\begin{array}{l}215(56.7) \\
164(43.3) \\
\end{array}$ & $\begin{array}{c}223(76.1) \\
70(23.9)\end{array}$ \\
\hline $\begin{array}{l}\text { Education level } \\
<\text { Ordinary level }(\%) \\
\text { Above Ordinary level }(\%)\end{array}$ & $\begin{array}{l}124(32.7) \\
255(67.3) \\
\end{array}$ & $\begin{array}{c}64(21.8) \\
229(78.2) \\
\end{array}$ \\
\hline $\begin{array}{l}\text { Marital status } \\
\text { Single }(\%) \\
\text { Married }(\%) \\
\text { Divorced }(\%) \\
\text { Widowed }(\%) \\
\end{array}$ & $\begin{array}{c}38(10.0) \\
312(82.3) \\
29(7.7) \\
9(2.4) \\
\end{array}$ & $\begin{array}{c}12(4.1) \\
267(91.1) \\
9(3.1) \\
4(1.4)\end{array}$ \\
\hline $\begin{array}{l}\text { Religion } \\
\text { Mainline }(\%) \\
\text { Protestants }(\%) \\
\text { Mapostori }(\%) \\
\text { Other }(\%) \\
\end{array}$ & $\begin{array}{c}76(20.0) \\
93(24.5) \\
273(72.0) \\
30(7.9) \\
\end{array}$ & $\begin{array}{c}54(18.4) \\
107(36.5) \\
97(33.1) \\
34(11.6) \\
\end{array}$ \\
\hline $\begin{array}{l}\text { Source of Health Education } \\
\text { VHW }(\%) \\
\text { EHT }(\%) \\
\text { Clinic }(\%) \\
\text { Other }(\%)\end{array}$ & $\begin{array}{c}114(32.2) \\
0(0.0) \\
247(65.2) \\
0(0.0) \\
\end{array}$ & $\begin{array}{c}97(33.1) \\
8(2.7) \\
133(45.4) \\
7(2.4) \\
\end{array}$ \\
\hline
\end{tabular}

Results at baseline presented in table 2, revealed that there was a statistically significant difference between the intervention and the control arm with regards to prevalence of child morbidity. The intervention arm had significantly higher child morbidity rates $(\mathrm{p}<0.001)$.

\begin{tabular}{|c|c|c|c|c|}
\hline $\begin{array}{l}\text { Variable } \\
(n=672)\end{array}$ & $\begin{array}{l}\text { Control sites } \\
(\mathrm{n}=273)(\%)\end{array}$ & $\begin{array}{l}\text { Intervention } \\
\text { sites } \\
(\mathrm{n}=379)(\%)\end{array}$ & Total & $\begin{array}{c}\text { Chi- } \\
\text { square } \\
\text { p-value }\end{array}$ \\
\hline $\begin{array}{l}\text { Pneumonia } \\
\text { No } \\
\text { Yes- }\end{array}$ & $\begin{array}{c}273(92.8) \\
20(7.2) \\
\end{array}$ & $\begin{array}{c}317(83.6) \\
62(16.4) \\
\end{array}$ & $\begin{array}{c}590(87.8) \\
82(12.2)\end{array}$ & $<0.001 *$ \\
\hline $\begin{array}{l}\text { Diarrhoea } \\
\text { No } \\
\text { Yes } \\
\end{array}$ & $\begin{array}{c}262(89.4) \\
31(10.6) \\
\end{array}$ & $\begin{array}{l}281(74.1) \\
98(25.9) \\
\end{array}$ & $\begin{array}{l}543(80.8) \\
129(19.2) \\
\end{array}$ & $<0.001 *$ \\
\hline $\begin{array}{l}\text { Malaria } \\
\text { No } \\
\text { Yes } \\
\end{array}$ & $\begin{array}{c}293(100.0) \\
0(0.0) \\
\end{array}$ & $\begin{array}{c}373(98.4) \\
6(1.6) \\
\end{array}$ & $\begin{array}{c}667(99.3) \\
5(0.7) \\
\end{array}$ & $<0.001 *$ \\
\hline $\begin{array}{l}\text { Malnutrition } \\
\text { No } \\
\text { Yes } \\
\end{array}$ & $\begin{array}{c}291(99.3) \\
2(0.7) \\
\end{array}$ & $\begin{array}{c}377(99.2) \\
2(0.8) \\
\end{array}$ & $\begin{array}{c}668(99.4) \\
4(0.6) \\
\end{array}$ & 0.955 \\
\hline $\begin{array}{l}\text { Anaemia } \\
\text { No } \\
\text { Yes } \\
\end{array}$ & $\begin{array}{c}288(99.3) \\
5(0.7) \\
\end{array}$ & $\begin{array}{c}375(98.4) \\
4(1.6) \\
\end{array}$ & $\begin{array}{c}663(98.7) \\
9(1.3) \\
\end{array}$ & 0.584 \\
\hline $\begin{array}{l}\text { Overall } \\
\text { morbidity } \\
\text { No } \\
\text { Yes }\end{array}$ & $\begin{array}{c}233(79.6) \\
60(20.4)\end{array}$ & $\begin{array}{l}212(55.9) \\
167(44.1)\end{array}$ & $\begin{array}{l}445(66.2) \\
227(33.8)\end{array}$ & $<0.001 *$ \\
\hline
\end{tabular}

*Asterisk represents statistically significant differences.

\section{Follow-up phase: Results and analysis}

Overall, the cumulative total number of educational sessions conducted during the twelve months of study implementation was 1704 . Of this total, two-thirds of the educational sessions (1137) were conducted in the intervention arm. The highest number of sessions was recorded in the Murewa clusters. The cumulative total number of home visits conducted during the twelve months of study implementation was 6861. Of this total, about twothirds of the home visits (4726) were conducted in the intervention arm. The highest number of home visits was recorded in Murewa clusters.

Community maternal and child healthcare topics covered during the twelve months of follow-up in study clusters were recorded. A cumulative total of 461 topics were reported in the intervention arm whereas 357 topics were covered in the control.

Intervention dosage was measured by assessing the number of community mobilisation meetings, the number of home visits and the number of people reached by the intervention in the intervention arm and the control arm. Table 3 below shows the number of home visits, mobilization meetings conducted per month in each of the four health centres that participated in this study and numbers of people reached.

TABLE 3: INTERVENTION DOSAGE BY HEALTH FACILITY AND STUDY ARM

\begin{tabular}{c|c|c|c|c|c}
\hline \multicolumn{3}{c|}{ Intervention arm } & \multicolumn{3}{c}{ Control arm } \\
\hline Home visits & Mean & SD & Home visits & Mean & SD \\
\hline Macheke & 393.8 & 25.3 & Marirangwe & 143.7 & 92.3 \\
\hline Murewa & 306.3 & 71.6 & Kunaka & 69.8 & 21.7 \\
\hline $\begin{array}{c}\text { Intervention } \\
\text { overall }\end{array}$ & 337.6 & 65.1 & $\begin{array}{c}\text { Control } \\
\text { overall }\end{array}$ & 133.4 & 95.6 \\
\hline $\begin{array}{c}\text { Mobilisation } \\
\text { meetings }\end{array}$ & Mean & $\mathrm{SD}$ & $\begin{array}{c}\text { Mobilisation } \\
\text { meetings }\end{array}$ & Mean & $\mathrm{SD}$ \\
\hline Macheke & 92.0 & 8.7 & Marirangwe & 36.1 & 4.9 \\
\hline Murewa & 75.2 & 4.3 & Kunaka & 34.3 & 2.2 \\
\hline $\begin{array}{c}\text { Intervention } \\
\text { overall }\end{array}$ & 81.2 & 8.8 & $\begin{array}{c}\text { Control } \\
\text { overall }\end{array}$ & 35.4 & 6.1 \\
\hline $\begin{array}{c}\text { People } \\
\text { reached }\end{array}$ & Mean & $\mathrm{SD}$ & $\begin{array}{c}\text { People } \\
\text { reached }\end{array}$ & Mean & $\mathrm{SD}$ \\
\hline Macheke & 436.80 & 14.6 & Marirangwe & $\begin{array}{c}\text { Median } \\
400\end{array}$ & $\begin{array}{c}\mathrm{IQR} \\
300\end{array}$ \\
\hline Murewa & 345.8 & 37.5 & Kunaka & 219.0 & 51.5 \\
\hline $\begin{array}{c}\text { Intervention } \\
\text { overall }\end{array}$ & 441.3 & 41.0 & $\begin{array}{c}\text { Control } \\
\text { Overall }\end{array}$ & $\begin{array}{c}\text { Median } \\
472\end{array}$ & $\begin{array}{c}\text { IQR } \\
230\end{array}$ \\
\hline
\end{tabular}

The Table 4 below summarises the analysis of results on the intensity of mobilisation activities in each study arm. These are presented in two different dimensions. The first dimension was just the mean number of topics covered each month cumulatively over the twelve months of study even where topics might be repeat topics. However, the second dimension compares the mean number of different (new topics) covered in the follow-up period.

TABLE 4: INTENSITY OF MOBILISATION ACTIVITIES BY STUDY ARM

\begin{tabular}{l|c|c|l|l|c}
\hline \multicolumn{2}{l|}{\begin{tabular}{l} 
Intervention arm \\
\hline $\begin{array}{l}\text { Mean number of topics } \\
\text { covered }\end{array}$
\end{tabular}} & SD & $\begin{array}{l}\text { Mean number of topics } \\
\text { covered }\end{array}$ & SD \\
\hline Macheke & 32.4 & 3.8 & Marirangwe & 20.9 & 5.2 \\
\hline Murewa & 33.2 & 5.3 & Kunaka & 24.6 & 1.3 \\
\hline $\begin{array}{l}\text { Intervention } \\
\text { overall }\end{array}$ & 32.9 & 5.7 & Control overall & 22.3 & 8.9 \\
\hline $\begin{array}{l}\text { Mean number of differential } \\
\text { topics covered }\end{array}$ & $\mathrm{SD}$ & $\begin{array}{l}\text { Mean number of differential } \\
\text { topics covered }\end{array}$ & $\mathrm{SD}$ \\
\hline Macheke & 15.2 & 3.8 & Marirangwe & 6.6 & 1.4 \\
\hline Murewa & 14.8 & 3.6 & Kunaka & 7.6 & 1.8 \\
\hline
\end{tabular}

One-year incidence of childhood illnesses (Pneumonia; Diarrhoea; Malaria; Fever; Anaemia; Malnutrition) were compared in the two study arms. Inferences were applied at 
the individual level while randomization was at the cluster or group level. The unit of randomization was therefore different from the unit of analysis. In this study, the incidence of child morbidity was measured over the twelve months of followup.

Fig. 4 is a summary of the disease cases that were reported in the intervention arm and the control arm over the 12 months of follow-up.

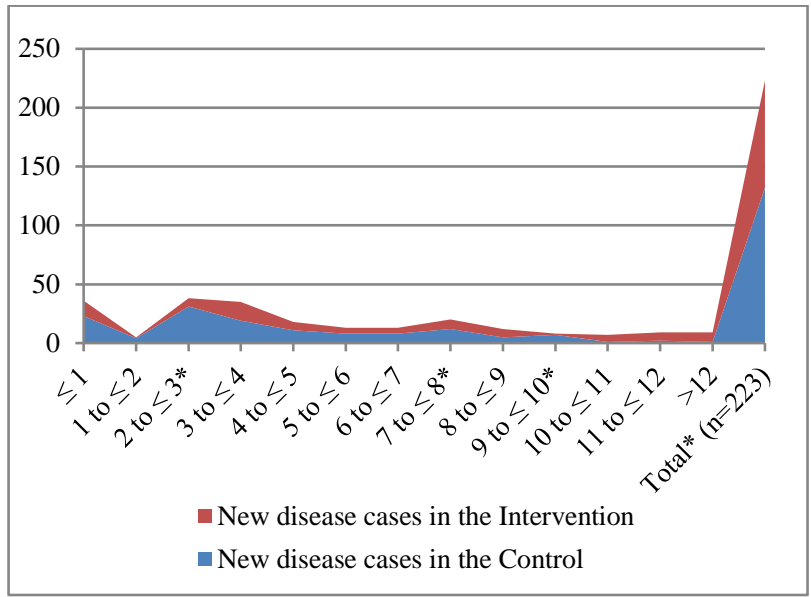

Fig. 4. All new disease cases by month.

Table 5 below summarises the relative risk ratio and incidence rate ratio per arm per month by cluster and study arm. The results in table 5 reveal that 91 cases of childhood illnesses were reported in the intervention and 132 cases in the control. The difference in number of cases between the intervention and the control arm was significant $(\mathrm{p}<0.001)$.

TABLE 5: RELATIVE RISK RATIO / INCIDENCE RATE RATIO OF DISEASE CASES IN THE CONTROL VS. INTERVENTION ARM

\begin{tabular}{c|c|c|c}
\multicolumn{4}{c}{ CASES IN THE CONTROL VS. INTERVENTION ARM } \\
\hline Cases & Intervention & Control & Overall \\
\hline Non-cases & 322 & 132 & 223 \\
\hline Risk & 0.22 & 220 & 542 \\
\hline $\begin{array}{c}\text { Risk } \\
\text { Difference } \\
\text { (95\% C.I) }\end{array}$ & & 0.36 & 0.29 \\
\hline $\begin{array}{c}\text { Risk Ratio } \\
\text { (95\% C.I) }\end{array}$ & & & 0.16 \\
\hline $\begin{array}{c}\text { Incidence Rate } \\
\text { Incidence Rate } \\
\text { Ratio } \\
\text { (95\% C.I) }\end{array}$ & 0.022 & 0.043 & $(0.09-0.22)$ \\
\hline
\end{tabular}

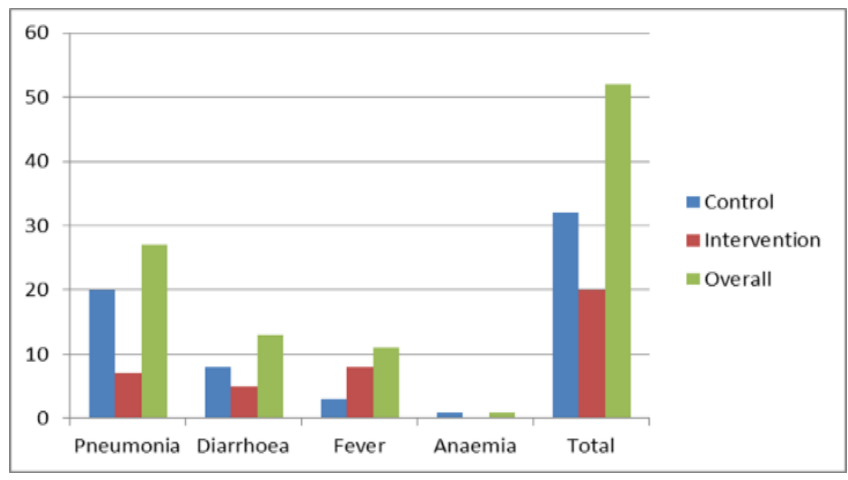

Figure 5. Repeat cases by condition and study arm

Fig. 6. shows the number of repeat cases by condition and arm. The mean number of repeat cases for Kunaka Clinic was
1.1 (SD 0.9), 2.5 (SD 2.0) for Marirangwe Clinic, 0.5 (SD 0.3 ) for Murewa Clinic and 0.9 (SD 0.9) for Macheke Clinic.

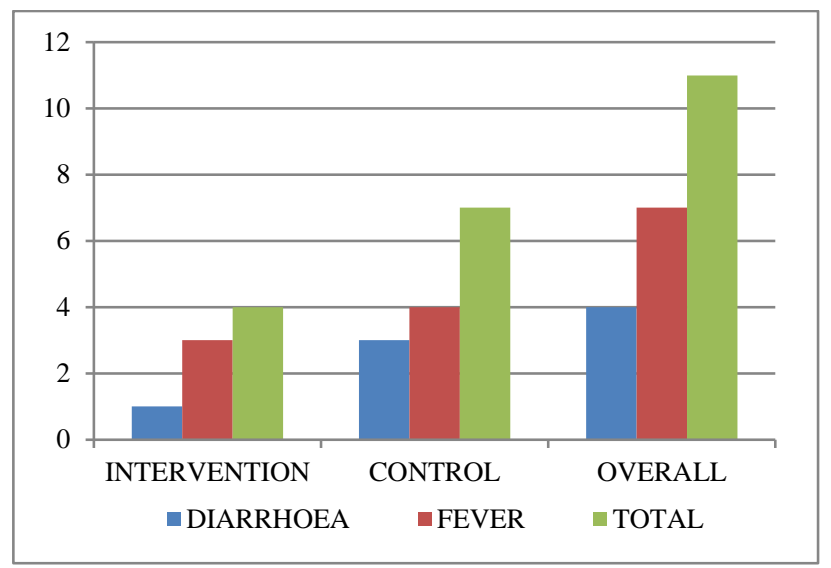

Fig. .6. Severe cases by condition and arm.

\section{Adjusted analysis for possible confounders and clustering}

Overall, there were 51(14.5\%) HIV positive children in the control arm against $25(6.1 \%)$ in the intervention arm, thus the relative risk was $1.679(0.823 ; 3.421)$ and this was not statistically significant, $\mathrm{p}=0.150$. Generalized linear models were used to determine the effect of HIV status, period in months, the cluster distance from the clinic and the cluster size on child morbidity. Unknown HIV status significantly increased the odds of contracting disease 14.4 times when adjusted for other known HIV statuses, period of follow-up in months, the cluster distance from the clinic, the cluster size and number of participants per cluster (AOR 14.4; $\mathrm{p}<0.001$ ). The distance to the clinic $(\mathrm{p}=0.936)$, period in months $(\mathrm{p}=0.319)$ and the cluster size $(\mathrm{p}=0.258)$ had no statistically significant effect on the disease outcome.

\section{DISCUSSION}

The study sought to determine the effectiveness of the ICM in reducing child morbidity through a cluster randomised controlled trial. At baseline, there was a significantly higher prevalence rate of child morbidity in the intervention arm when compared with the prevalence rate in the control arm. At the end of the follow-up period, results showed a statistically significant reduction in child morbidity. The study hypothesized that the ICM would reduce child morbidity and this hypothesis was supported. The ICM reduced the incidence of child morbidity by addressing social and behavioral determinants of health and healthcare seeking behaviour.

The overall risk of child morbidity was $37.5 \%$ in the control arm and $22.0 \%$ in the intervention arm. This meant that the risk of contracting diseases in the control arm was 1.7 times $(p<0.001)$ higher than that in the intervention arm. The incidence of being a disease case without intervention was increased by 1.96 times $(\mathrm{p}<0.001)$ compared to when the intervention is done. When compared to baseline findings on overall morbidity, the intervention reduced child morbidity by $22.1 \%$, from $44.1 \%$ to $22.0 \%$, whereas child morbidity increased by $17.1 \%$ in the control arm.

Similar studies on child morbidity have revealed that 
effective community intervention approaches can reduce child morbidity. A quasi-experimental study $(\mathrm{N}=300)$ conducted in San Luis reduced the incidence of diarrhoeal diseases among children aged $0-59$ months by $18 \%$ between survey rounds [6].

Unknown HIV status significantly increased the odds of contracting other diseases 14.4 times when adjusted for other known HIV statuses, period of follow up in months, cluster distance from the clinic, household size and number of participants per cluster. This means that to reduce child morbidity, governments may need to make HIV testing mandatory for all pregnant women and for children underfive so that they are put on treatment if eligible. Being HIVpositive but on treatment was protective against other childhood illnesses.

A study in Malawi revealed that morbidity rate amongst HIV-exposed children was 1.34 times higher $(\mathrm{p}<0.001)$ as compared to children not exposed [7], [8]. HIV exposure at birth has been associated with increased child morbidity and mortality, especially before the first birthday.

The effect of distance was not significant, and showed no consistent pattern. The cluster effects were therefore inconsistent with distance but the interventions effect. This result differs from what was observed in Tanzania on determinants of care seeking for children under-five years, which reported that there was twice (95\% CI: 1.11-2.72) as likely to be a delay in taking children living more than $5 \mathrm{~km}$ from the nearest health facility to receive medical attention than there was for those living at shorter distances from these facilities [7], [9]. Similarly, in Uganda, the most significant challenge in utilizing services was long distance to health facilities [8].

The study only used the IMNCI guidelines to assess childhood illnesses and confirm diagnosis. For disease conditions such as anaemia future studies could consider using more robust methods of evaluating anaemia such as biomarkers so that more accurate incidences and prevalence can be determined. In this study, inferences were made at individual level while randomization was at cluster level. The application of standard statistical methods generally tends to bias p-values downwards leading to spurious statistical significance (Type 1 error) [7], [10]. However, in this study, clustering was accounted for in both the estimation of trial size and in the analysis to address the potential and common problems in cluster randomised trials. Selection of districts could have been improved by considering districts with similar population profiles. Seke is more urban than Murewa which could also have impacted on child morbidity, given the differences in HIV prevalence and exposure rates and also the variation in the epidemiology of disease conditions like malaria and other seasonal diseases.

The study therefore concludes that the integration of the ICG approach into the main health delivery system will ensure the consistent transference of correct knowledge and skills for better maternal and child health. This happens through the perpetuation of a cycle of continuous health education, monitoring and promotion.

Such an intervention will improve both maternal and child health outcomes by reducing the incidence of maternal and child morbidity. The Government of Zimbabwe and other developing countries should consider implementing such an approach in their respective community health strategies in order to reduce preventable child morbidity and mortality.

The study design being a cluster randomised controlled trial could not completely answer the questions as to why people adopt different stances on healthcare even when they are exposed to the same knowledge about healthcare practices. A study design that combines the qualitative and quantitative aspects would add more information useful for policy-makers.).

\section{ACKNOWLEDGMENT}

I would like to begin by expressing my sincere gratitude to Professor Mathilda Zvinavashe, Professor Lovemore Gwanzura and Professor Babill-Stray Pedersen for all the support, technical guidance and encouragement that they provided throughout this study journey. I would also like to extend my gratitude to the NORHED for the financial support throughout the course of my study. I could not have made it without this support and the opportunities received to interact with and learn from other members of the NORHED consortium.

\section{REFERENCES}

[1] L. Li, O. Shefali, H. Daniel, P. Jamie, C. Simon, and M. Colin, "Global, regional, and national causes of child mortality in 2000-13, with projections to inform post-2015 priorities: an updated systematic analysis", The Lancet. 14. 61698-6, 2015.

[2] UNICEF. (2015) Under-five mortality. Retrieved from: https://data.unicef.org/topic/child-survival/under-five-mortality.

[3] Zimbabwe National Statistics Agency. Zimbabwe Perinatal Audit Report. 2nd edn. Harare. Zimbabwe National Statistics Agency, 2009.

[4] Zimbabwe National Statistics Agency and ICF International. Zimbabwe Demographic and Health Survey 2010-11. Final Report. ICF International, Inc. Calverton, Maryland USA. Zimbabwe National Statistics Agency (ZIMSTAT) and ICF International. 2012.

[5] Zimbabwe National Statistics Agency. Multiple Indicator Cluster Survey. Final report. Harare. Zimbabwe National Statistics Agency. (2014).

[6] T. Harkins, C. Drasbek, J. Arroyo, and M. McQuestion, "The health benefits of social mobilisation: experiences with community-based Integrated Management of Childhood Illness in Chao, Peru and San Luis, Honduras", Promot Educ, 15(2), 15-20. 2008.

[7] O. Divala, C. Michelo, and B. Ngwira, "Morbidity and mortality in HIV-exposed under-five children in a rural Malawi setting: a cohort study", J Int AIDS Soc, 17, 19696. 2014.

[8] T. Kassile, R. Lokina, P. Mujinja, and B.P. Mmbando, "Determinants of delay in care seeking among children under five with fever in Dodoma region, central Tanzania: a cross-sectional study", Malar J, 3; $13,348,2014$.

[9] D. Musoke, P. Boynton, C. Butler, and M.B. Musoke, "Health-seeking behaviour and challenges in utilizing health facilities in Wakiso district, Uganda" Afr Health Sci, 14(4), 1046-55, 2014.

[10] H. Seokyung, P. Suezann, J.T. David, and W. Judith, "Methodological bias in cluster randomised trials", BMC Med Res Methodol, 5, 10. 2005 . 